\title{
LA SOSTENIBILIDAD DE LAS PENSIONES PÚBLICAS EN ESPAÑA: ASPECTOS BÁSICOS
}

\section{PUBLIC PENSIONS SUSTAINABILITY IN SPAIN: BASIC ISSUES}

\section{José M. Domínguez Martínez}

Catedrático de Hacienda Pública

Universidad de Málaga

jmdominguez@uma.es ORCID 0000-0001-6859-2717

Recepción de trabajo: 03-06-2021 - Aceptación: 08-06-2021

- I. INTRODUCCIÓN. $\square$ II. Sistemas públicos de pensiones de reparto: esquema de flujos y variables condicionantes. - III. EL SISTEMA PÚBLICO DE PENSIONES EN ESPAÑA: ESCENARIO DEMOGRÁFICO, ECONÓMICO Y FINANCIERO. — IV. CONCLUSIONES. — V. BIBLIOGRAFÍA 


\section{RESUMEN}

En este trabajo se lleva a cabo un análisis introductorio del sistema público de pensiones de España desde el punto de vista de la sostenibilidad. Inicialmente se expone el esquema de flujos y variables que condicionan la sostenibilidad de un sistema de reparto. A continuación, partiendo de la situación presupuestaria actual, se examinan las proyecciones demográficas y financieras, las cuales arrojan un escenario de déficits estructurales en las décadas venideras. Finalmente se esbozan diversas líneas de reforma para hacer frente a dicha situación.

PALABRAS CLAVE: Pensiones, Sostenibilidad, Sistema de pensiones de reparto, Déficit Seguridad Social, España.

\section{ABSTRACT}

The aim of this work is to develop an introductory analysis of the Spanish public pension system from a sustainability point of view. Firstly, the scheme of flows and variables that condition the sustainability of an unfunded pension system is reviewed. Next, taking the current budgetary situation as a reference, the demographic and financial projections -resulting in structural deficits along coming decades- are under scrutiny. Finally, several paths of reform so as to face that imbalance are adressed.

KEYWORDS: Pensions, Sustainability, Unfunded pension system, Social Security deficit, Spain. 


\section{INTRODUCCIÓN}

La sostenibilidad es un concepto clave en el campo de las finanzas públicas. Con carácter general, existe sostenibilidad de las finanzas públicas cuando el conjunto de los ingresos públicos no financieros permite hacer frente, de manera estable a lo largo del tiempo, a los gastos públicos, tanto los de naturaleza —en sentido económico — no financiera como financiera. Dicho de otra manera, el sistema no será sostenible si se genera un déficit presupuestario estructural de cuantía relevante de manera permanente.

Esta noción es aplicable a cualquier ámbito del sector público, con una perspectiva institucional o territorial. La Seguridad Social no constituye, por tanto, ninguna excepción al respecto. Sin perjuicio de ello, por lo que respecta al sistema de pensiones, la sostenibilidad presupuestaria se encuentra subordinada a la sostenibilidad actuarial. Difícilmente podrá garantizarse la primera si se prescinde de la segunda.

En España está vigente un sistema público de pensiones de reparto, que arrastra desde hace años importantes problemas de sostenibilidad, especialmente puestos de manifiesto a raíz de las consecuencias de la denominada "Gran Recesión", y posteriormente agravados en el contexto de la crisis provocada por la pandemia del coronavirus.

Un sistema de pensiones de reparto es un sistema que desafía la lógica económica y que fía su continuidad a la progresiva incorporación de cohortes al mercado de trabajo con altas tasas de ocupación. Por su propia configuración, al carecer de una consistencia económica intrínseca, se muestra altamente vulnerable al juego de variables económicas y demográficas que pueden ocasionar agudos desequilibrios entre los flujos de entrada y de salida de recursos. A partir de un sistema en funcionamiento, basado en unas expectativas por parte de los cotizantes y en compromisos con los beneficiarios, resulta sumamente complicado arbitrar soluciones que requieran ajustes severos de las aportaciones y, singularmente, de las prestaciones. Más allá de los impactos en las economías familiares, la relevancia de los componentes asociados al mercado político introduce dificultades adicionales. La accidentada trayectoria de las reformas y de los planteamientos de revisión de esquemas en España acredita sobradamente los escollos que han de afrontarse.

En este trabajo se lleva a cabo un análisis introductorio del sistema público de pensiones de España desde el punto de vista de la sostenibilidad. Inicialmente se expone el esquema de flujos y variables que condicionan la sostenibilidad de un sistema de reparto. A continuación, partiendo de la situación presupuestaria actual, se examinan las proyecciones demográficas y financieras, las cuales arrojan un escenario de déficits estructurales en las décadas venideras. Finalmente se esbozan diversas líneas de reforma para hacer frente a dicha situación. 


\section{SISTEMAS PÚBLICOS DE PENSIONES DE REPARTO: ESQUEMA DE FLUJOS Y VARIABLES CONDICIONANTES}

Los sistemas de pensiones basados en el reparto, al igual que los de capitalización, presentan una serie de ventajas e inconvenientes'. El funcionamiento de un sistema de pensiones de reparto responde a la interacción de un amplio conjunto de factores y variables que afectan tanto a los ingresos obtenidos a partir de las cotizaciones sociales como al montante de las prestaciones a satisfacer. Los más relevantes y significativos aparecen reflejados en el esquema 1.

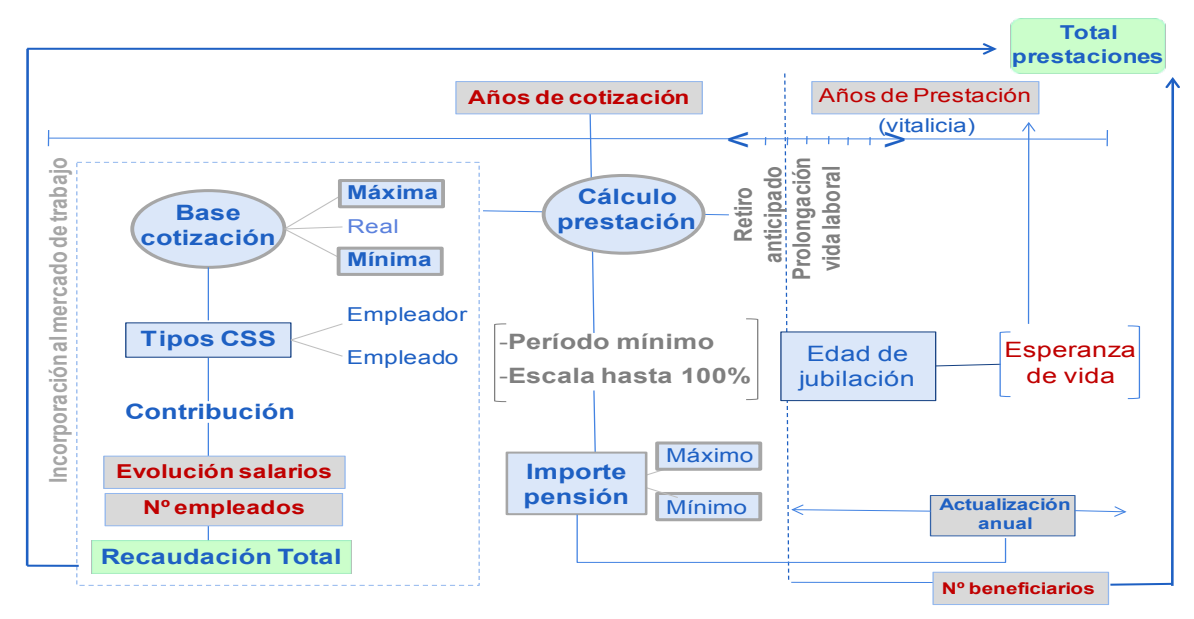

Esquema 1: Variables básicas de un sistema de pensiones de reparto. Fuente: Domínguez Martínez (2012)

El principal riesgo de un sistema de estas características es el de la posible insostenibilidad financiera. La condición Aaron-Samuelson demuestra que, en un sistema de reparto, la tasa de rendimiento fiscalmente sostenible es la suma del crecimiento de la productividad (o ingresos medios) y el crecimiento (o contracción) de la fuerza laboral ${ }^{2}$. No obstante, Barr y Diamond (2009) arguyen que el problema financiero no es inherente a un sistema de prestación definida, ya que puede ser diseñado para ajustarse automáticamente a las variables demográficas.

En el otro extremo, hay quienes sostienen que el sistema de reparto tiene algunas connotaciones propias de los sistemas piramidales ${ }^{3}$. En cualquier caso, no hay que perder de vista que los sistemas

1 Una sintesis se ofrece en Domínguez Martínez (2012, pág. 3).

2 Vid. Disney (1999, pág. 21).

3 Vid., por ejemplo, Calzada (2010), y The Economist (2011), donde se afirma que "la seguridad social sigue un esquema de pirámide: los trabajadores futuros han de generar los ingresos impositivos para pagar las prestaciones de los futuros pensionistas y esto es un problema si una generación es más pequeña que la anterior". Goetzmann (2017, págs. 264-265) identifica en los sistemas de pensiones de reparto el mismo problema financiero de raíz que se dio en Francia y Gran Bretaña, en los siglos XVII y XVIII, con la venta por los gobiernos de anualidades vitalicias. 
LA SOSTENIBILIDAD DE LAS PENSIONES PÚBLICAS EN ESPAÑA: ASPECTOS BÁSICOS

de pensiones constituyen un terreno especialmente abonado para el mantenimiento de falacias y la posibilidad de errores analíticos (Domínguez Martínez, 2012). El incumplimiento de la regla de Tinbergen, al atribuir a un mismo instrumento la consecución de diferentes objetivos — de seguro y redistributivo - introduce una patente dificultad tanto en el plano analítico como a la hora de aportar soluciones eficaces y transparentes ${ }^{4}$.

La encrucijada en la que se encuentran los sistemas de pensiones de reparto viene ilustrada por la condición de equilibrio financiero (esquema 2). Se trata de una ecuación bastante simple en términos agregados: cuando disminuye la ratio empleados/pensionistas, solo hay tres alternativas: a) aumentar los salarios; b) aumentar el tipo de las cotizaciones; c) disminuir las prestaciones.

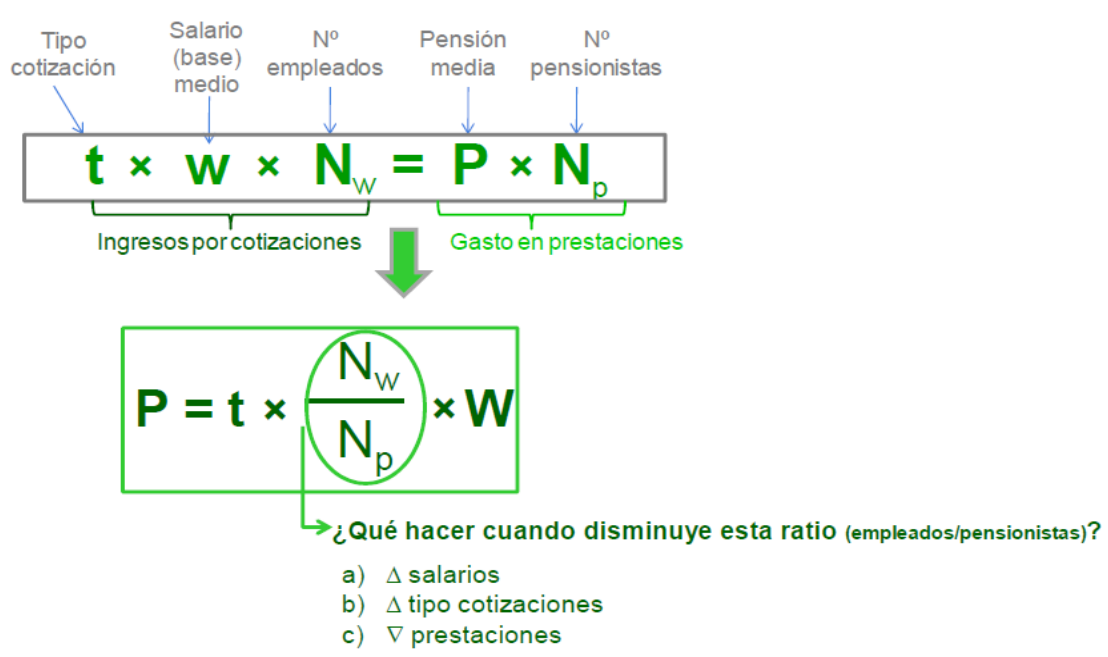

Esquema 2: Sistema de pensiones de reparto: condición de equilibrio financiero. Fuente: Domínguez Martínez (2012)

Como apunta Doménech (2019a), la condición de equilibrio entre ingresos y gastos implica que la pensión media sobre el salario medio (tasa de prestación) debe ser igual al producto de los ingresos totales sobre los salarios de la economía por la relación entre cotizantes por pensión.

El proceso de envejecimiento poblacional tiene consecuencias importantes en el plano económico, sobre la oferta y la demanda agregadas, así como sobre las políticas macroeconómicas (Banco de España, 2019, pág. 227).

Adicionalmente, las crisis económicas impactan de lleno en el esquema anterior. La reciente crisis provocada por la pandemia del coronavirus ha afectado a los sistemas de pensiones, con carácter general, a través de diversos canales. Los principales son ${ }^{5}$ : i) una mayor probabilidad de que haya indivi- 
LA SOSTENIBILIDAD DE LAS PENSIONES PÚBLICAS EN ESPAÑA: ASPECTOS BÁSICOS

duos que abandonen el mercado de trabajo y soliciten el cobro de pensiones; ii) la disminución de las bases de cotización agregadas como consecuencia de la contracción del empleo y de salarios reales estancados o decrecientes; iii) shocks de precios de activos con impacto en los balances de los sistemas de capitalización; y iv) capacidad de gobiernos y empresas, como titulares de obligaciones de pensiones, para mantener la solvencia de los sistemas de prestación definida bajo condiciones adversas.

\section{EL SISTEMA PÚBLICO DE PENSIONES EN ESPAÑA: ESCENARIO DEMOGRÁFICO, ECONÓMICO Y FINANCIERO}

El gráfico 1 refleja la evolución de las pensiones contributivas y de las cotizaciones sociales en España (Hernández de Cos, 2021; Arce, 2019a; 2019b). En él se aprecia cómo, después de una larga etapa en la que la recaudación por cotizaciones sociales superaba el montante de las pensiones, desde 2012 se abre una brecha en sentido contrario. 2020 ha sido el noveno año consecutivo con desequilibrio del saldo presupuestario no financiero del sistema de pensiones. Como destaca Hernández de Cos (2021), el incremento del gasto de las pensiones contributivas ha superado con creces el leve aumento de los ingresos por cotizaciones sociales. En los últimos años, el déficit del sistema de pensiones roza el 3\% del PIB.

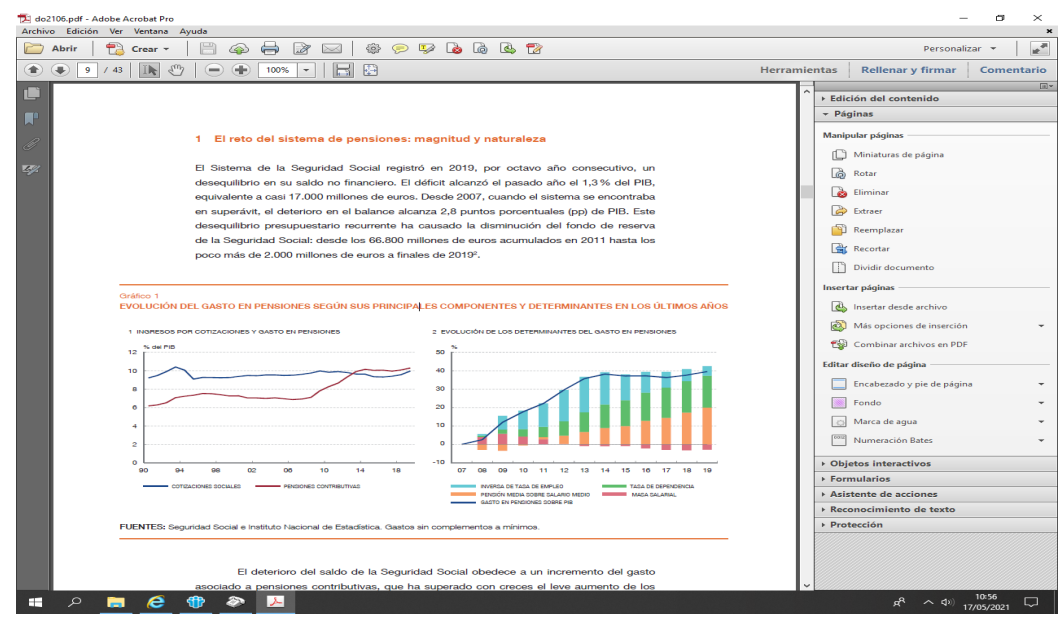

Gráfico 1: Evolución de los ingresos por cotizaciones y del gasto en pensiones. Fuente: Hernández de Cos (2021)

Diversos estudios e informes se han ocupado de efectuar proyecciones de las cuentas económicas del sistema público de pensiones en el horizonte de mediados del siglo XXI ${ }^{6}$. El modelo desarro-

6 El panorama de los años venideros inmediatos se analiza por Devesa y Doménech (2021b). También puede verse Doménech (2019b). Para el año 2024 se prevé un déficit de unos 35.000 millones de euros, equivalente al 2,5\% del PIB. 
LA SOSTENIBILIDAD DE LAS PENSIONES PÚBLICAS EN ESPAÑA: ASPECTOS BÁSICOS

Ilado por la AIReF (Pastor y Vila, 2019) se basa en las interrelaciones de tres módulos (demográfico, mercado de trabajo y pensiones). Según las previsiones realizadas, de una ratio de gasto público en pensiones sobre el PIB del 10,6\% en 2018 se pasaría a un 13,4\% en 2048, cifra que se elevaría hasta el 16,9\% si no se aplicaran las reformas aprobadas en 2011 y 2013.

En AIReF (2020) se efectúa una actualización de las proyecciones realizadas por este organismo en 2019, que apunta a una ratio gasto público en pensiones/PIB del 14,2\% en el año 2050 (escenario central) (gráfico 2).

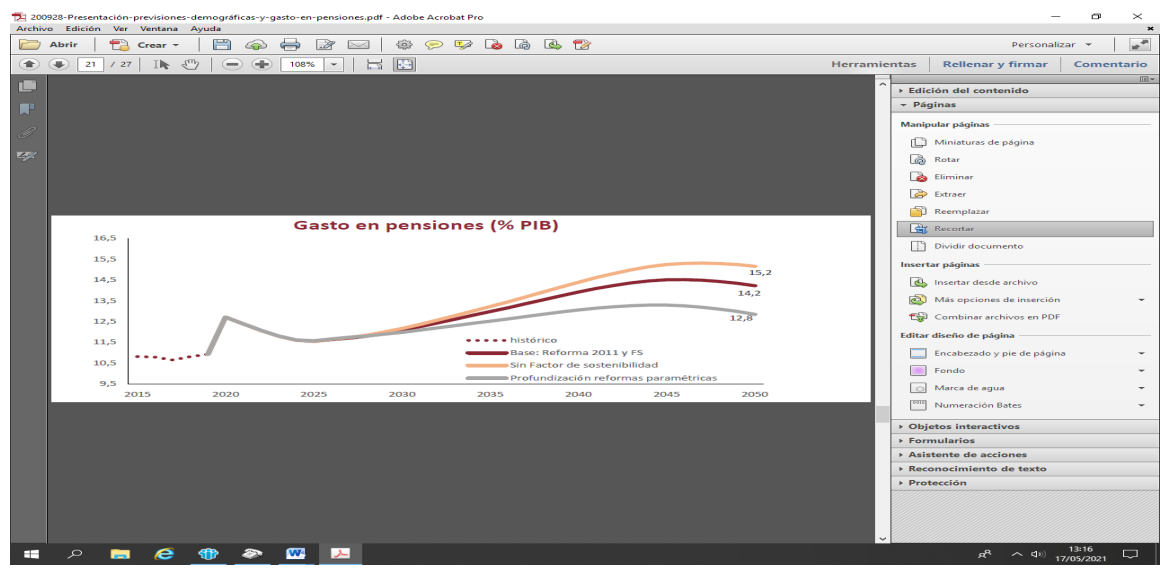

Gráfico 2: Proyecciones del gasto público en pensiones. Fuente: AIReF (2020)

La Comisión Europea ha publicado proyecciones del gasto público en el horizonte 2070. Según dichas proyecciones, la ratio gasto público en pensiones/PIB pasará del 12,3\% en 2019 al 13,2\% en 2045, y al 10,3\% en 2070 (European Commission, 2021, pág. 68). No puede perderse de vista la dinámica de los factores determinantes del gasto que subyacen a dicha evolución prevista7 . Es destacable que la relación entre la pensión media y el salario medio de la economía pasaría del 66\% en 2019 al 29\% en 2070 (European Commission, 2021, pág. 80). Para el año 2050, se prevé una ratio de gasto del 13,0\% y unas contribuciones sociales equivalentes al 11,8\% del PIB (European Commission, 2021, pag. 301) . $^{2}$

Por su parte, la Comisión de Seguimiento y Evaluación de los Acuerdos del Pacto de Toledo (2020) ha elaborado un amplio informe en el que se expresa un rechazo contundente a la sustitución del sistema de reparto por otro de capitalización (2020, págs. 61 y 75), y se formula una veintena de recomendaciones, después de declarar que "se ha construido, mantenido y desarrollado una obra gigantesca, fruto de un acto colectivo de reflexión previsora" (2020, pág. 37). Uno de los aspectos clave del informe es la tesis de que las cotizaciones sociales han servido para sustentar otras políticas del

7 Vid. Ministerio de Asuntos Económicos y Transformación Digital (2021).

8 Las cotizaciones sociales representaron en 2019 el 11,8\% del PIB. Esta cifra se mantiene constante en las previsions a lo largo del tiempo. Vid. Ministerio de Asuntos Económicos y Transformación Digital (2021, pág. 34). 
LA SOSTENIBILIDAD DE LAS PENSIONES PÚBLICAS EN ESPAÑA: ASPECTOS BÁSICOS

Estado (2020, pág. 62), por lo que, mediante el trasvase de ciertas partidas, se solventaría la situación de desequilibrio en el sistema de pensiones ${ }^{\text {. }}$.

Sin embargo, diversos analistas, como De la Fuente et al. (2020), consideran que tanto el informe del Pacto de Toledo como los representantes del Gobierno de la Nación comparten "un diagnóstico francamente optimista e intencionadamente tranquilizador"10. Así, de los gastos de la Seguridad Social considerados como impropios en el informe del Pacto de Toledo (2020) (22.871 millones de euros), sólo una parte (4.702 millones de euros) podrían catalogarse como tales (De la Fuente et al., 2020, pág. 5). Además de la insuficiencia de la recaudación procedente de las cotizaciones sociales, la indexación completa a la inflación es muy costosa y las perspectivas demográficas, sombrías (De la Fuente et al., 2020, pág. 5; Hernández de Cos, 2021).

\section{LA SOSTENIBILIDAD DEL SISTEMA PÚBLICO DE PENSIONES EN ESPAÑA: LÍNEAS DE REFORMA}

De manera sigilosa, a lo largo de los últimos años, el déficit presupuestario de la Seguridad Social en España ha ido reptando hasta llegar a representar una parte muy significativa del déficit del conjunto de las Administraciones Públicas"1. Con una perspectiva temporal más amplia, aunque, a fuerza de ser repetida, la noción de "bomba de relojería" se había convertido en un tópico aparentemente inocuo, ahora empieza a tomar cuerpo como seria amenaza.

La equidad actuarial es uno de los elementos clave para poder calibrar la sostenibilidad de un sistema de pensiones. Se trata de un rasgo primordial de cualquier sistema de seguro.

Diversos estudios y análisis revelan que el sistema de pensiones en España, en términos globales, no se encuentra equilibrado desde ese punto de vista. Así, por ejemplo, según el Instituto de Actuarios Españoles (2020), "la arquitectura actual del sistema contributivo de pensiones de jubilación en España implica que, en la gran mayoría de los casos, el pensionista recibe más de lo que ha aportado en su vida laboral, financiándose la diferencia a través de impuestos o de más deuda pública". La magnitud del factor de equidad actuarial estimada en este informe se cifra en 1,51, lo que viene a significar que el sistema contributivo de jubilación devuelve un 51\% más de lo que recibe.

Evidentemente, el déficit se trasladaría al conjunto del sector público.

10 El Pacto de Toledo sigue siendo una potente ancla en el debate sobre el sistema de pensiones. Según el Banco de España (2010), dicho Pacto, aprobado en 1994, "en general... fue valorado en su momento muy positivamente por la mayor parte de los analistas, aunque más en términos de su alcance como acuerdo político que como instrumento práctico válido a largo plazo, dada la falta de su concreción y las ambigüedades de algunas de sus recomendaciones". El caso español respalda plenamente el aserto de Martin y Whitehouse (2008) cuando afirmaban que la reforma de las pensiones es el "tercer rail" de la política: "tócalo y morirás". 
LA SOSTENIBILIDAD DE LAS PENSIONES PÚBLICAS EN ESPAÑA: ASPECTOS BÁSICOS

Por otro lado, según un estudio del Banco de España (Moraga y Ramos, 2020), el sistema otorga, en media, en torno a 1,7 euros de prestación por cada euro aportado. Los resultados varían según los supuestos considerados, pero los aspectos básicos tienden a confirmarse. Según nuestras propias estimaciones (Domínguez Martínez, 2020), podrían llegar a obtenerse 2,4 euros por cada euro de cotización. La relación sería de 1 a 1 si el pensionista vive algo más de 9 años a partir de la jubilación. Si lo longevidad es inferior, percibirá menos de 1 euro por cada euro aportado.

La falta de transparencia y la transmisión de unas percepciones irreales son una barrera de primer orden para la puesta en marcha de cualquier proceso de reforma. Así, se ha apuntado que los beneficiarios se resisten a recortes en las prestaciones, creyendo que han pagado ya por tales derechos, incluso aunque el valor de las prestaciones pueda exceder significativamente del valor de sus contribuciones (Domínguez Martínez, 2011) ${ }^{12}$.

A pesar de que la esperanza de vida es la medida biométrica más relevante en el ámbito de las pensiones, en España no se ha incorporado adecuadamente en el diseño del sistema de pensiones públicas (Ayuso, 2021).

En este contexto, debido a la relativa generosidad de nuestras pensiones en comparación con los niveles salariales, España gasta 2 puntos porcentuales respecto al PIB más que un país típico de la Unión Europea (De la Fuente et al., 2020)13.

Algunos analistas han abogado por la aplicación de la denominada "regla de oro" como inspiradora de la reforma del sistema de pensiones. Dicha regla consiste en tratar de lograr un equilibrio entre los costes y los beneficios para las generaciones presentes y futuras (Cardoso, 2021).

Si tenemos en cuenta la evolución real y prevista de la ratio de dependencia (población con 65 años o más respecto a la población entre 15 y 64 años), que ha pasado del 20\% en 1990 al 30\% en 2018 (32,1\% en 2019), y se estima que llegará al 60\% en 2050 (62,5\% en 2070) (Eurostat, 2019, pág. 9; 2021, pág. 19; European Comission, 2021, pág. 23), el reto para el sistema de pensiones es más que evidente ${ }^{14}$. Además, a raíz del aumento de la longevidad, se estima que la duración del período de jubilación pasará de 20,7 (hombres) y 23,9 (mujeres) años en 2019 a 23,3 y 26,7, respectivamente (European Commission, 2021, pág. 61).

El Informe del Pacto de Toledo (2020) basa su estrategia en el principio de separación de fuentes $^{15}$. Aun cuando, como se ha señalado, algunas partidas, por su naturaleza, podrían excluirse del ámbito de la Seguridad Social, no todas las seleccionadas corresponden a "gastos impropios" de la Seguridad Social (De la Fuente et al., 2020, pág. 5).

12 Éste no es un problema exclusivo de España. Vid. Stiglitz (2000, pág. 363).

13 La tasa de prestación es del 58\% del salario medio, y la de reposición, del $80 \%$.

14 La ratio de los mayores de 65 años respecto a las personas con edad entre 20 y 64 años se prevé que sea prácticamente del $80 \%$ en el año 2050 . Vid. 0 CDE (2021, pág. 38).

15 Una valoración de las recomendaciones del Pacto de Toledo se efectúa en Devesa y Doménech (2020). 
LA SOSTENIBILIDAD DE LAS PENSIONES PÚBLICAS EN ESPAÑA: ASPECTOS BÁSICOS

La AIReF (2020), en línea con lo señalado, ha expuesto la necesidad de llevar a cabo reformas paramétricas: retrasar la edad efectiva de jubilación, ampliar la carrera de cotización, e incluir un factor de sostenibilidad. Sin embargo, hay que tener presente que dos importantes elementos de la reforma de 2013 han sido suspendidos desde 2018, el factor de sostenibilidad (ajuste del nivel inicial de la pensión en función de los cambios en la esperanza de vida), y la actualización de las pensiones según un índice basado en el saldo estructural del sistema de pensiones, en lugar del IPC (OCDE, 2021, pág. 38).

A tenor del panorama descrito, resulta difícil no suscribir la constatación de la OCDE (2021, pág. 39) cuando afirma que "es crucial que medidas adecuadas y socialmente aceptables para asegurar la sostenibilidad financiera del sistema de pensiones se adopten rápidamente".

Ante el problema de sostenibilidad, son varias las alternativas que pueden plantearse (Hernández de Cos et al., 2017; Devesa y Doménech, 2021a): a) traspasar todo el ajuste sobre los contribuyentes futuros; b) ajustar las pensiones vigentes con revalorizaciones inferiores al crecimiento del nivel de precios; c) implantar un sistema de reparto de cuentas nocionales ${ }^{16}$. Devesa y Doménech (2021a) ponen de relieve algo tan elemental como frecuentemente soslayado: "un sistema de pensiones de reparto no garantiza a priori la cuantía exacta de las futuras pensiones ni a los pensionistas actuales ni a los futuros", lo que está ligado al hecho de que el principio en el que se basa el supuesto "contrato intergeneracional" de que el sistema garantiza un esfuerzo similar de cada generación no necesariamente se traduce en una tasa de reemplazo igual. La condición recogida en el esquema 2 nos recuerda cuáles son las opciones básicas existentes.

La reforma del sistema de pensiones debería reunir una serie de características a fin de asegurar la sostenibilidad (Devesa y Doménech, 2021a): i) que garantice el equilibrio actuarial y financiero del sistema; ii) que el equilibrio financiero se garantice mediante un mecanismo automático de ajuste; iii) ante ajustes discrecionales, que sea gradual; iv) que sea percibida como justa; v) que sea transparente; vi) que permita una flexibilidad en la edad de jubilación. En este contexto, el sistema de cuentas nocionales individuales es una fórmula que contiene elementos interesantes que pueden contribuir a facilitar la sostenibilidad del sistema y a mejorar su transparencia. Este sistema, mediante elementos de contribución definida, permitiría aquilatar el montante de los compromisos asumidos. Se lleva una contabilidad de las cotizaciones a lo largo de la vida laboral, a las que se atribuye una rentabilidad. Llegado el momento de la jubilación, el importe así determinado se convierte en una renta vitalicia, en términos de equivalencia actuarial. No obstante, el sistema seguiría siendo de reparto ${ }^{17}$.

En suma, al margen de reformas paramétricas, es necesario realizar alguna reforma de corte estructural, como se ha indicado. Hay muchas opiniones al respecto, pero no faltan quienes abogan por rediseñar el sistema articulándolo en tres pilares: i) prestaciones mínimas financiadas a través 
LA SOSTENIBILIDAD DE LAS PENSIONES PÚBLICAS EN ESPAÑA: ASPECTOS BÁSICOS

de impuestos para quienes no hayan podido acceder al segundo pilar; ii) pilar contributivo, respecto al que caben distintas opciones, como la introducción de un enfoque de aportación definida; iii) bloque complementario basado en los planes de pensiones privados, de empleo e individuales (Domínguez Martínez, 2018).

El grado de viabilidad del sistema de pensiones puede analizarse a partir de distintos enfoques metodológicos y con una mayor o menor sofisticación. Sin embargo, como ha recordado Hernández de Cos (2021), existen básicamente dos alternativas para garantizar la sostenibilidad financiera de dicho sistema: aumentar los recursos que llegan a él, o disminuir la generosidad de las prestaciones (o bien, mediante una combinación de ambas). Las dos variables constituyen los determinantes últimos, si bien, como se ha señalado anteriormente, son múltiples los factores subyacentes en cada caso.

Según ha expresado Hernández de Cos (2021, pág. 18), el trade off prestación vs esfuerzo de financiación tiende a deteriorarse: "A efectos ilustrativos, si se asumen una tasa de empleo del $80 \%$ y un factor demográfico del 58\% en 2050, sería necesario un aumento del tipo efectivo de las cotizaciones sociales de 8 pp, hasta el 32\% (o un aumento equivalente en otros impuestos y su transferencia al sistema de pensiones), para mantener constante la tasa de beneficio actual, o, alternativamente, la tasa de beneficio debería caer hasta el 34\%, desde el 46\% actual, para mantener constante el tipo efectivo". En definitiva, "la disyuntiva entre tasa de beneficio y contribuciones al sistema de pensiones será más desfavorable que en el pasado" (Arce, 2021, pág. 12).

En principio, como ocurre con cualquier proceso basado en flujos de ingresos y gastos, podrían adoptarse dos enfoques: i) cuantificar los desembolsos asumibles y determinar los gastos que podrían atenderse de manera estable y sostenida; ii) establecer el montante de las prestaciones que se pretende satisfacer, y buscar los recursos necesarios para poder garantizarlas.

Lo ideal sería que el sistema fuese diseñado con una coherencia interna y que, a lo largo del tiempo, pudiera desempeñar eficazmente su cometido mediante, en su caso, la puesta en marcha de mecanismos de ajuste automático cuyos efectos fuesen conocidos de antemano. Indudablemente, la concepción de un sistema que se basara en un cambio periódico de las reglas del juego, en detrimento de la situación de los participantes, o en un traslado de la carga al conjunto de los contribuyentes, no requeriría de demasiadas dotes hacendísticas.

\section{CONCLUSIONES}

El equilibrio financiero a largo plazo es una condición imprescindible para la supervivencia de un sistema de pensiones. Hace años, Stiglitz (2000, págs. 380-381) ponía de relieve una cuestión económica fundamental: “¿Puede el gobierno hacer ajustes relativamente menores al programa de pensiones para lograr una viabilidad financiera a largo plazo?" 
LA SOSTENIBILIDAD DE LAS PENSIONES PÚBLICAS EN ESPAÑA: ASPECTOS BÁSICOS

Si no puede, según el Premio Nobel de Economía, las restricciones presupuestarias estrictas impuestas por la privatización podrían ser la única vía de salida del laberinto político en el que los problemas son continuamente trasladados de una generación a otra.

En España resulta imprescindible adoptar una serie de medidas que permitan encontrar una salida estructural que no ponga en peligro un pilar tan importante de la cohesión social.

Durante años, la sociedad española vivió amparada en la creencia de unos sólidos pilares económicos. Si hasta hace relativamente poco cualquier manifestación que arrojara alguna duda acerca de la sostenibilidad del sistema de pensiones era casi una garantía de acusación de herejía, los déficits crecientes de las cuentas de la Seguridad Social desde el año 2012 han transformado las inquietudes en preocupaciones palpables. Y aunque están siendo los pensionistas actuales quienes más abiertamente muestran su descontento, es la capacidad del sistema para hacer frente a medio plazo a sus compromisos la que constituye el núcleo del problema.

Según los distintos escenarios económicos explorados, en ausencia de un incremento de los ingresos del sistema, se tendría que producir una disminución de la magnitud relativa de las prestaciones (importe de las pensiones respecto al salario medio) para asegurar la sostenibilidad del sistema de pensiones.

En un sistema de pensiones de reparto, mientras los flujos de entrada sean iguales o superiores a los de salida, no se presenta problema alguno; sin embargo, cuando los primeros se frenan (insuficiente ritmo de creación de empleo, evolución lenta de los salarios) o se amplían los segundos (mayor período de percepción de prestaciones por la mayor longevidad, importe superior de las pensiones entrantes en comparación con las salientes), aparecerá un desequilibrio. La inversión de la pirámide demográfica española, unida a otros factores, representa un reto extraordinario.

El sistema de reparto rompe el vínculo directo entre lo aportado durante la vida laboral y lo percibido en la etapa de jubilación, y establece una prestación definida vitalicia calculada en función de las bases de cotización de parte de la vida activa. Aparentemente, la mayoría de los pensionistas presentes y futuros aspira a que el sistema sea justo con ellos. Una interpretación razonable de la justicia en el ámbito previsional es que la suma actualizada de todas las aportaciones efectuadas se equipare a la suma, también actualizada, del conjunto de las prestaciones recibidas. Pues bien, a pesar de la percepción generalizada acerca de la supuesta injusticia del sistema, en la práctica, éste es generoso para aquellas personas que perciban prestaciones durante un período no inferior a unos nueve años.

La sostenibilidad de un sistema de pensiones de reparto depende del mantenimiento de un equilibrio entre los ingresos y los gastos. Básicamente, los primeros dependen de las siguientes variables: empleo, salarios, economía sumergida, años de cotización, bases de cotización y tipos de cotización; los segundos, de estas otras: edad de jubilación, importe de la pensión inicial, criterio de evolución de la pensión anual y longevidad.

Por un cúmulo de circunstancias, el sistema de pensiones se encuentra en la actualidad en un atolladero. Para lograr encontrar la senda liberadora y no tomar falsos atajos es preciso desplegar 
LA SOSTENIBILIDAD DE LAS PENSIONES PÚBLICAS EN ESPAÑA: ASPECTOS BÁSICOS

un plan de actuación en varios frentes: i) una política económica orientada al crecimiento sostenible e inclusivo; ii) fomento de la innovación y la productividad; iii) lucha contra el fraude y la economía sumergida; iv) aplicación de ajustes paramétricos a fin de adecuar la cuantía de los compromisos por pensiones a los recursos generados para su cobertura; v) incorporación de aportaciones extraordinarias y transitorias para cubrir los déficits, mediante alternativas que no penalicen el empleo; vi) estudio de la implementación de una reforma estructural que permita, mediante elementos de contribución definida, como en el sistema de cuentas nocionales, aquilatar el montante de los compromisos asumidos.

Las pensiones tienen importantes componentes políticos, sociales y psicológicos, pero la viabilidad del sistema ha de descansar en bases económicas. Sin percibir claramente cuál es la esencia de éstas seguiremos atrapados en un laberinto. Para intentar localizar la salida es necesario primero eliminar los espejos deformantes que impiden orientarnos.

\section{BIBLIOGRAFÍA}

AIReF (2020): “Actualización de previsiones demográficas y de gasto en pensiones”. Documento Técnico 1/20.

Arce, Ó. (2019a): “Presentación”, en V Jornada Planes de Pensiones en España. Panel I. Pensiones y ahorro: presente y soluciones de futuro, El País Economía-Cinco Días, 19 de noviembre.

Arce, Ó. (2019b): “Los retos del envejecimiento poblacional para la política fiscal”, Banco de España, 29 de mayo.

Arce, Ó. (2021): "Algunas consecuencias económicas de los cambios demográficos”, Banco de España, 27 de mayo

Ayuso, M. (2021): “El necesario enfoque actuarial de los sistemas de pensiones: la relevancia de la esperanza de vida, también en España", Fedea, Documento de Trabajo 2021/01.

Banco de España (2010): "La reforma del sistema de pensiones en España".

Banco de España (2019): “Consecuencias económicas de los cambios demográficos”, en "Informe Anual 2018”.

Barr, N., y Diamond, P. A. (2009): “Reforming pensions: principles, analytical errors and policy directions”, International Social Security Review, vol. $62, \mathrm{n}^{\circ} 2$.

Calzada, G. (2010): “Ocho mitos sobre la reforma de las pensiones”, Expansión, 24 de septiembre.

Cardoso, M. (2021): “La regla de oro”, Expansión, 23 de enero.

Comisión de Seguimiento y Evaluación de los Acuerdos del Pacto de Toledo (2020): “Informe de evaluación y reforma del Pacto de Toledo”, Boletín Oficial de las Cortes Generales, Congreso de los Diputados, Serie D, núm. 175, 10 de noviembre.

De la Fuente, Á.; García Díaz, M. Á.; y Sánchez Martín, A. R. (2020): “Algunas reflexiones sobre el informe del Pacto de Toledo y los planes del Gobierno en materia de pensiones", Fedea Policy Papers, 2020/3. 


\section{LA SOSTENIBILIDAD DE LAS PENSIONES PÚBLICAS EN ESPAÑA: ASPECTOS BÁSICOS}

Devesa, E., y Doménech, R. (2020): “Sostenibilidad de las pensiones: casi todo por hacer”, Vozpópuli, 5 de noviembre.

Devesa, E., y Doménech, R. (2021a): “Las cuentas nocionales individuales: elemento central de la reforma del sistema de pensiones en España", Fedea Policy Papers, 2021/02.

Devesa, E., y Doménech, R. (2021b): “El déficit de pensiones en tiempo de reforma”, Vozpópuli, 7 de mayo.

Disney, R. (1999): “Notional accounts as a pension reform strategy: an evaluation”, SP Discussion Paper, No. 9928, The World Bank. Doménech, R. (2019a): “En busca del equilibrio en el sistema de pensiones”, Vozpópuli, 18 de marzo.

Doménech, R. (2019b): “El sistema público de pensiones en España: proyecciones y perspectivas de mejora”, BBVA Research, 12 de enero.

Domínguez Martínez, J. M. (2011): “¿Es rentable invertir en pensiones públicas?”, La Opinión de Málaga, 15 de junio.

Domínguez Martínez, J. M. (2012): “La reforma de las pensiones en España: una aproximación económica”, Instituto de Análisis Económico y Social, Universidad de Alcalá, Documentos de Trabajo 02/2012.

Domínguez Martínez, J. M. (2018): “Las pensiones y el doctor Pangloss”, diario Sur, 13 de mayo.

Domínguez Martínez, J. M. (2020): “Acerca de la rentabilidad de las pensiones públicas”, diario Sur, 13 de septiembre.

European Commission (2018): "The 2018 Ageing Report. Economic \& Budgetary Projections for the 28 EU Member States (20162070)", European Economy, Institutional Paper 079.

European Commission (2021): "The 2021 Ageing Report. Economic \& Budgetary Projections for the EU Member States (20192070)", European Economy, Institutional Paper 148.

Eurostat (2021): "Ageing Europe. Looking at the lives of older people in the EU. 2019 edition".

FMI (2020): “Pension schemes in the COVID-19 crisis: impact and policy considerations”, Fiscal Affairs, 20 de julio.

Goetzmann, W. N. (2017): "Money changes everything. How finance made civilization possible”, Princeton University Press.

Grupo de Investigación en Pensiones y Protección Social (2021): "El déficit contributivo anual del sistema de la Seguridad Social sube al $2,75 \%$ del PIB, mientras que el déficit por operaciones no financieras baja al 1,34\% tras el cierre previsional de $2020 "$, http://www.uv.es/pensiones.

Hernández de Cos, P.; Gimeno, J. F., y Ramos, R. (2017): “El sistema público de pensiones en España: situación actual, retos y alternativas de reforma", Documentos Ocasionales № 1701, Banco de España.

Hernández de Cos, P. (2021): “El sistema de pensiones en España: una actualización tras el impacto de la pandemia. Contribución del Banco de España a los trabajos de la Comisión de Seguimiento y Evaluación de los Acuerdos del Pacto de Toledo. 2 de septiembre de 2020", Banco de España, Documentos Ocasionales, № 2106.

Instituto de Actuarios Españoles (2020): "Factor de equidad actuarial del sistema contributivo de pensiones de jubilación español", 27 de agosto.

Martin, J. P., y Whitehouse, E. (2008): “Reforming retirement-income system: lessons from the recent experiences of OECD countries", OECD Social, Employment and Migration Working Papers, $n^{\circ} 66$. 


\section{LA SOSTENIBILIDAD DE LAS PENSIONES PÚBLICAS EN ESPAÑA: ASPECTOS BÁSICOS}

Ministerio de Asuntos Económicos y Transformación Digital (2021): “Ageing Report 2021. Country fiche Spain”, en Eurostat Commission (2021).

OCDE (2021): "OECD Economic Surveys: Spain”, mayo.

Moraga, M, y Ramos, R, (2020): “Una estimación del rendimiento financiero del sistema de pensiones”, Boletín Económico 3/2020.

Pastor, Á., y Vila, M. (2019): "Modelo AIReF de proyección del gasto en pensiones en España”, Serie Documentos de Trabajo AIReF, DT/2019/1.

Sáez de Jáuregui, L. M. (2020): “Una dulce, pero intensa, reforma de las pensiones, Expansión, 2 de septiembre.

Stiglitz, J.E. (2000): "Economics of the public sector”, Norton.

The Economist (2011): "Pensions, Ponzis and Pyramids", 24 de septiembre.

Valero, D.; Artis, M.; Ayuso M., y García, J. (2011): “Una propuesta de reforma del sistema de pensiones español basada en un modelo de contribución definida nocional”, Revista de Métodos cuantitativos para la Economía y la Empresa, junio.

Wachs, D., y Onrubia, J. (2021): "Automatic adjustment mechanisms in public pension reforms: effects over fiscal sustainability, adequacy, and fairness", Fedea, Documento de Trabajo, 2021/05. 